\title{
Histoire du français et sa présence actuelle dans le royaume thaï
}

\section{Patchareerat Yanaprasart}

\section{(2) OpenEdition \\ 1 Journals}

\section{Édition électronique}

URL : https://journals.openedition.org/dhfles/2056

DOI : 10.4000/dhfles.2056

ISSN : 2221-4038

Éditeur

Société Internationale pour l'Histoire du Français Langue Étrangère ou Seconde

Édition imprimée

Date de publication : 1 juin 2001

Pagination : 45-72

ISSN : 0992-7654

\section{Référence électronique}

Patchareerat Yanaprasart, " Histoire du français et sa présence actuelle dans le royaume thaï »,

Documents pour l'histoire du français langue étrangère ou seconde [En ligne], 26 | 2001, mis en ligne le 14 septembre 2017, consulté le 27 mai 2021. URL : http://journals.openedition.org/dhfles/2056 ; DOI :

https://doi.org/10.4000/dhfles.2056

Ce document a été généré automatiquement le 27 mai 2021.

(C) SIHFLES 


\title{
Histoire du français et sa présence actuelle dans le royaume thaï
}

\author{
Patchareerat Yanaprasart
}

\section{Présence française dans le royaume de Siam}

1 En 1985, la France et le royaume de Thaïlande ont célébré le tricentenaire de leurs relations diplomatiques, ouvertes à l'époque de Louis XIV et du roi Naraï. Ainsi, la présence de la langue française en Thaïlande ne date pas d'hier. Au XVIIe siècle déjà, des responsables du royaume de Siam avaient fait l'effort d'apprendre le français pour mieux communiquer avec les envoyés du roi français.

2 Cette esquisse historique des premières relations diplomatiques à la fin du XVIIe siècle a pour but de mettre en valeur les profonds liens entre ces deux nations et leur coopération bilatéralement développée tout au long des 338 années (1662-2001), en particulier dans la promotion de la langue de Voltaire dans le pays des Thaïs.

\subsection{Relations diplomatiques franco-thaïes ${ }^{1}$}

3 Les rapports diplomatiques et commerciaux entre la France et la Thaïlande débutèrent à l'époque du roi Naraï du Siam (1666-1688) et du roi de France Louis XIV (1643-1715). Au royaume d'Ayutthaya (ancienne capitale du Siam), l'année 1662 vit l'arrivée de la première équipe de missionnaires jésuites français, dirigée par Mgr de la Motte Lambert et l'évêque de Berytus, et chargée de deux lettres pour le roi du Siam dont l'une de Louis XIV et l'autre du pape Clément IX. Conscient du dessein de Louis XIV de convertir le souverain au catholicisme, le Siam réserva pourtant un accueil privilégié aux représentants de la Cour française. Quant au roi Naraï, il accorda aux jésuites français des terres où ils édifièrent des écoles et des églises.

Ces jésuites jouèrent un rôle déterminant dans les relations franco-siamoises. Sept ans après leur débarquement au Siam, une relation d'ordre diplomatique entre les deux pays se renforçait en 1680, quand le premier diplomate thaïlandais Phya Pipatkosa 
embarqua pour la France dans l'objectif d'établir des liens d'amitié avec la cour française; il sombra en mer en 1681.

5 Sur le plan commercial, Louis XIV sollicita la protection du roi Naraï pour les marchands français en envoyant une petite mission commerciale en 1682, en relation avec la Compagnie française des Indes orientales, qui avait ouvert en 1681 son premier comptoir sur les rives de la Ménam Chao Praya, non loin de Bangkok.

Désireux de nouer des contacts avec les Français, le roi Naraï envoya en 1684 à Paris une deuxième ambassade siamoise, sous la direction de Khun Pijaiwanit et Khun Pijitmaitri, pour demander l'envoi d'une mission diplomatique française à Ayutthaya et la signature d'un traité d'alliance.

7 Selon la volonté du roi de France de convertir le roi Naraï et son peuple au catholicisme, l'ambassadeur français (le chevalier de Chaumont) accompagné de l'abbé de Choisy arriva au Siam en 1685 avec deux bateaux de guerre. De son côté, le Siam profita du retour de cette mission à Paris en 1686 pour envoyer une délégation thaïe conduite par Phraya Kosathibodi (Pan).

8 Le XVIIe siècle représenta l'ouverture de la Thaillande sur l'éducation et le commerce avec l'extérieur. Le français, langue d'élite, fut introduit pour la première fois à la cour royale thaïe et devint une langue étrangère largement utilisée dans le cadre du commerce et de la politique. En 1687 une seconde ambassade française arriva au Siam, et plusieurs événements eurent lieu cette année-là. Le début de 1687 vit le stationnement des troupes françaises à Bangkok contre les ambitions anglaises et hollandaises ; suivit la création d'une base militaire française sur la côte est de la baie du Bengale afin de favoriser le commerce franco-thaï. Celle-ci fut complétée par deux autres débarquements à Ayutthaya : l'un de Claude Cébéret du Boullay, le directeur de la Compagnie française des Indes orientales, et l'autre de Simon de la Loubère. Une mission débarqua ensuite au Siam, comprenant six cent trente-six soldats, des diplomates et des jésuites transportés dans des bateaux de guerre. En décembre, la France et le Siam conclurent un traité commercial, qui garantissait essentiellement des privilèges et des facilités commerciales surtout en ce qui concerne l'extraterritorialité des employés français, le monopole de l'étain à Phuket et la possession des îles près d'Ayutthaya.

9 Au fil de leur installation, les Français établis dans le royaume entrèrent en conflit et se divisèrent en différentes factions. La mort de Naraï, en 1688, entraîna le retour au pouvoir des conservateurs, qui expulsèrent les Européens. En raison de l'appréhension ressentie à l'égard de la christianisation et du mécontentement vis-à-vis des comportements des missionnaires français, un sentiment antifrançais se répandit à la cour du nouveau souverain. Suite à une réaction de la nouvelle équipe dirigeante contre la politique profrançaise du régime précédent, les Français durent s'embarquer précipitamment. Quelques jours après la signature du traité de commerce, Cébéret quitta Ayutthaya et La Loubère, qui dut écrire un livre sur les relations historiques avec le Siam, repartit dans des circonstances peu agréables le 3 janvier 1688. Le Siam se ferma à l'Occident pendant deux siècles dès 1688 , la fin du règne de Naraï.

10 Au XIXe siècle, les interventions de l'Occident en Indochine placèrent le Siam dans une situation difficile dès le règne de Rama III (1824-1851). Le renouvellement des relations diplomatiques entre la France et la Thaillande se réalisa, sous le règne de Napoléon III (1852-1870) et du roi Mongkut (ou Rama IV 1851-1868). La réinstallation de l'Ambassade de France, la construction d'églises, d'écoles catholiques et la signature d'accords 
politiques et commerciaux s'effectuèrent en 1856. Avec l'avènement du roi Mongkut, doué d'un esprit encyclopédique et passionné d'astronomie, apparurent les prémices de la modernité. Ce roi promut à nouveau une politique d'échanges commerciaux et culturels avec l'Occident. Dès son arrivée au pouvoir, il établit des relations diplomatiques avec l'Europe et les États-Unis. Pendant le règne de ce monarque éclairé, les entraves au commerce furent levées, d'importants traités furent négociés et signés avec de nombreuses puissances européennes et les sciences modernes furent introduites. Le fait d'avoir été éduqué en anglais et en latin par des savants occidentaux a permis au Roi d'avoir la clairvoyance de comprendre en profondeur la culture occidentale. C'est à partir de cette période que commença la propagation des langues étrangères, parmi lesquelles l'anglais et le français, dans le territoire siamois.

11 La politique de modernisation du pays se poursuivit sous le règne du roi Chulalongkorn (ou Rama V 1868-1910). Cette politique d'ouverture aux cultures occidentales comme aux relations extérieures permit paradoxalement au royaume de préserver son indépendance par rapport aux pouvoirs colonisateurs occidentaux.

Cependant, l'augmentation de l'influence française en Indochine amena le Siam à abandonner à la France en 1887 sa souveraineté sur le sud-est du Tonkin. Après une série de négociations, le royaume fut contraint par la France à lui céder en 1893 la rive gauche du Mékong (une grande partie du Laos). 1907 fut l'année du traité de frontière franco-siamois selon lequel la Thaïlande renonça, au profit de la France, à ses droits sur les territoires riverains situés à l'ouest du Mékong (dans la province de Louang Prabang -le nord-ouest du Cambodge). Enfin, le nord de la Malaisie fut remis à la France en 1909. En échange de l'abandon par les Français de leurs privilèges d'extraterritorialité au Siam et de l'indépendance de certaines provinces frontalières siamoises, le Siam réussit ainsi à préserver son indépendance.

13 Après de tels incidents et conflits entre les deux pays, liés aux intérêts coloniaux français, les nuages se dissipèrent.

\subsection{Introduction \& évolution du français dans le système éducatif thaï}

$14 \mathrm{Au}$ XVIIe siècle, le roi Narai, qui entretenait de bonnes relations avec le roi de France, donna le terrain du district Mahapran au Chao Phya Wijayen-dra (Constantine Phaulkon) pour y faire construire l'école de français appelée « le Collège Constantien ». Pendant cette période, deux personnes jouèrent un rôle très important : l'abbé de Tarchard et l'abbé de Choisi furent les deux premiers enseignants de français en Thaïlande (1672). Mais, les cours n'étaient dispensés qu'à la cour royale et cette initiative ne dura pas longtemps. L'enseignement du français en Thaïlande fut ensuite suspendu par peur de la colonisation et de 1'évangélisation de la part des Français. D'ailleurs, la nation était en guerre avec des pays voisins.

Deux siècles plus tard sous le règne du roi Chulalongkorn (Rama V), le pays élabora des programmes d'enseignement destinés à un public plus large.

Dès cette époque-là, le français fut officiellement reconnu au Siam comme branche scolaire. Historiquement, c'est le Révérend Père Colombert qui fonda la première école dispensant le français dans le Royaume thaï. La tentative de contrebalancer l'influence grandissante de l'anglais dans le royaume fut marquée par l'arrivée successive de 
missionnaires français. Partant d'une initiative à motifs politiques, cette école devint le collège de l'Assomption en 1885. Plus tard deux autres collèges, Saint-Gabriel et SaintPaul, furent fondés par des prêtres catholiques ; le premier couvent Saint-Joseph s'ouvrit pour l'éducation des filles. Un autre objectif principal de ces écoles francothaïes au moment de leur fondation fut de convertir les Thaïlandais au christianisme. Jusqu'au milieu du XIXe siècle, le français n'était pour les Thaïlandais qu'une langue de prestige réservée aux gens de la haute société. La formation en français n'avait pas de valeur utilitaire dans la vie pratique, mais elle était perçue comme un emblème de haute classe, un signe de supériorité. Ce jugement est encore en vigueur de nos jours. Cette langue a l'honneur insigne d'avoir toujours été appréciée à la Cour. Sa Majesté le Roi Rama IX parle français avec passion, ainsi que trois autres princesses. Son Altesse Royale la Reine Mère et sa fille aînée sont francophones, ayant appris le français en Suisse, tandis que Son Altesse Royale la Princesse Héritière, fille du roi, l'a étudié en Thaïlande.

$\mathrm{Au}$ fur et à mesure que le temps passe, la pratique du français se répand de plus en plus parmi le public thaï. Au XXe siècle, le français a gagné en importance. Au début du siècle, il était une langue scientifique internationale choisie pour la rédaction d'informations historiques, pour les traductions de l'histoire artistique et archéologique du pays.

De manière à promouvoir plus largement le français actuel, en 1977, Son Altesse Royale la Princesse Galayani Vadhana Krom Luang Naradhi-was Rajanagrinda, sœur aînée de Sa Majesté le Roi Bhumipol Adulyadej, prit l'initiative de fonder l'Association thaïlandaise des professeurs de français. Le 25 novembre 1997, vingt ans après la création de l'A.T.P.F., S.A.R. la Princesse, présidente honoraire de cette association, reçut les insignes de commandeur de Tordre national du Mérite, en raison de son rôle éminent en faveur de l'enseignement du français en Thaïlande.

La langue française a trouvé les faveurs d'un large public, qui la considère comme une langue de grande culture. Grâce aux liens étroits et anciens entre la France et la Thaïlande, l'image impressionnante de la langue française, la possibilité de l'apprendre et d'en poursuivre l'étude sur place jusqu'au doctorat placent le français en deuxième position derrière l'anglais. De ce fait, le ministère de l'Éducation nationale fait l'effort de promouvoir cette langue non seulement au niveau linguistique, mais aussi au niveau social.

Les repères chronologiques ${ }^{2}$ qui suivent ont pour but principal de permettre de mieux déterminer le développement du français depuis son introduction dans le milieu éducatif thaï jusqu'à aujourd'hui.

1893: Début officiel de l'enseignement du français en Thaïlande

1928: Introduction du français dans le programme d'études de l'université

Chulalongkorn

1943: Étude du français en tant que matière principale, secondaire ou optionnelle

1946: Création d'une maîtrise de français à l'université Chulalongkorn

1955: Introduction officielle du français dans l'enseignement secondaire

1960: Le français comme matière à option au deuxième cycle du secondaire

1970: Ouverture de la première Alliance française à Bangkok 1972: Ouverture de

l'Alliance française de Chiang Mai 1977: Fondation de l'Association thaillandaise des

professeurs de français (A.T.P.F.)

1988: Ouverture de l'Alliance française de Phuket 
1989: Développement des cours de français du tourisme 1990: Français en option libre dans le secondaire 1991 : Introduction du français scientifique dans le système éducatif 1995: Ouverture du doctorat de langue et littérature françaises

\section{Politique d'enseignement des langues étrangères d'après les programmes scolaires du ministère de l'Éducation}

En ce qui concerne la politique linguistique et l'élaboration des programmes d'études durant les cent dernières années, il importe de décrire les finalités assignées à l'enseignement des langues étrangères de chaque programme d'études, pour approfondir notre compréhension du rôle joué par le ministère de l'Éducation et pour mettre en évidence la spécificité de certains objectifs éducatifs en vigueur dans le milieu thaï.

\subsection{Aperçu historique}

Le programme d'études de 1895 (Curriculum B.E. 2438) a constitué le premier programme du Royaume déterminant l'apprentissage de toutes les langues étrangères depuis Prayoke 3 Chan 1.

Le Programme d'études de 1898 (Curriculum B.E. 2441) réserve l'enseignement du français en quatre ans aux écoles de pages d'honneur, aux écoles de langue thaïe et aux écoles de langue anglaise et thaïe.

Le Programme d'études de 1907 (Curriculum B.E. 2450) ne parle pas de l'enseignement du français. L'accent est plutôt mis sur la langue anglaise.

Le Programme d'études de 1913 (Curriculum B.E. 2456) prévoit que les élèves du deuxième cycle du secondaire peuvent apprendre le français.

En 1917, la première université de Thaïlande (Université Chulalongkorn) est fondée. Sous le patronage royal et avec le support gouvernemental, la modernisation de l'éducation s'étend à toutes les classes sociales.

En 1928, le français est proposé aux étudiants de la faculté des Sciences et Lettres comme une matière à part entière. La formation exigeait alors la connaissance de deux langues étrangères au moins, à choisir entre l'anglais, le chinois, le français, l'allemand. Elles étaient soit matière principale, soit matière secondaire.

Le programme d'études supérieures en français avait été stabilisé bien avant l'enseignement secondaire qui a commencé en 1948. Selon les programmes nationaux du ministère de l'Éducation, dans toutes les écoles secondaires, l'élève de lettres devait choisir une autre langue étrangère comme matière à option : le pâli ${ }^{3}$, le français ou le chinois et ce en plus de l'anglais.

30 En 1955, il y a un changement dans le programme du niveau secondaire. Les langues étrangères, l'anglais obligatoire et une autre langue facultative (français, chinois, allemand pâli ou sanskrit) deviennent obligatoires. L'apprentissage de la deuxième langue étrangère est aussi ouvert aux élèves de sciences. 
31 En 1960, le ministère de l'Éducation évoque la nécessité d'apprendre une deuxième langue étrangère, soit le français, l'allemand le chinois ou le pâli. L'objectif était de pouvoir utiliser les langues dans le cadre du contact quotidien et d'activités professionnelles simples. Il s'agissait aussi d'avoir une base pour des études supérieures et une compréhension convenable de la culture des natifs de la langue apprise.

Le Programme d'études de 1975 (curriculum B.E. 2518) met l'accent sur l'acquisition d'une formation d'ordre professionnel et d'une connaissance suffisante pour exercer de futures carrières. Les nouveaux objectifs scolaires suivants sont ajoutés dans ce programme éducatif : être capable d'écouter, de parler, de lire et d'écrire une langue simple dans le contexte de la vie quotidienne.

\subsection{Structure}

Depuis les années 1950, la promotion de l'essor linguistique fait partie d'une politique ambitieuse visant à élever le niveau d'éducation du pays, à lui permettre de faire face à ses importants besoins en ressources humaines. La politique de l'enseignement des langues est déterminée par le ministère de l'Éducation, selon lequel les langues des pays entretenant des relations amicales avec la Thaïlande jouissent d'un statut égal. Au niveau de l'éducation préuniversitaire, le Centre des programmes d'études (Curriculum Development Centre) est responsable de la planification de la politique éducative en matière de langues. Sa tâche principale est d'élaborer des programmes d'études pour les écoles publiques et privées. Alors que la mise en œuvre des programmes est assurée par le Centre, la mise en application des directives ministérielles et le contrôle sont à la charge de l'inspectorat, bureau qui joue un rôle d'intermédiaire, voire de coordinateur entre le ministère et l'équipe pédagogique.

$\mathrm{Au}$ niveau de l'éducation supérieure, le Bureau des affaires universitaires joue un rôle indirect dans l'exécution de la politique linguistique, dans le contrôle et l'approbation, étant donné que chaque université publique thaïlandaise a une certaine autonomie administrative.

C'est en fonction des priorités linguistiques au niveau national que sont élaborés les programmes d'études. La politique linguistique nationale de 1978 définit deux catégories de langues vivantes en Thailande : la langue nationale et les langues étrangères. Cela dit, à part le thaï, langue aussi bien officielle que nationale, et les quatre dialectes régionaux et certaines langues minoritaires, les autres langues, à savoir l'anglais, le français, l'allemand, le japonais, le chinois, l'arabe, sont tous classés sous l'étiquette langues étrangères.

36 En principe, aucune langue étrangère n'est incluse dans le programme de scolarité obligatoire. D'après le Programme national d'études de 1987 (remanié en 1990), l'anglais a le même statut que toute autre langue étrangère, mais dans la pratique, il a la priorité $\mathrm{du}$ fait qu'il joue le rôle de langue étrangère importante dans l'administration, les affaires et les échanges internationaux. A vrai dire, le statut privilégié de l'anglais vient du fait que c'est la langue étrangère de communication internationale la plus fréquemment utilisée comme instrument d'accès à d'autres connaissances. Pour cela, le Programme d'études de 1960 autorise déjà l'enseignement de l'anglais comme matière obligatoire aussi bien au primaire qu'au secondaire.

Octobre 1976, marquant l'arrivée au pouvoir d'un gouvernement réactionnaire sous Thanin, a vu changer le statut de l'anglais dans les programmes d'études. Dans ce 
contexte très nationaliste, non seulement l'anglais subventionné par le ministère de l'Éducation, mais aussi d'autres langues étrangères, ont été déclassées par le Conseil national de l'éducation. Cette réforme a souligné le rôle primordial de la langue nationale, le thaï standard. Jusqu'en 1978, l'anglais et les autres langues n'étaient que des matières à option dispensées seulement au secondaire.

A partir de mars 1978, avec la nouvelle politique linguistique dans l'éducation, l'anglais n'est plus subventionné au niveau primaire. La plupart des écoles publiques, surtout à Bangkok, enseignent l'anglais aux élèves à partir de Prathom Suksa $5^{4}$ à raison de 5 séances au maximum par semaine ou selon le besoin local. Quant aux écoles privées où le besoin d'apprentissage de l'anglais se fait sentir et où les conditions matérielles sont favorables, l'anglais est proposé aux élèves comme matière hors programme, et ce dès leur première année primaire, c'est-à-dire à partir de Prathom Suksa 1.

L'enseignement secondaire ${ }^{5}$ dure 6 séances et se divise en deux cycles : le premier cycle (Matayom Suksa 1 à $3^{6}$ ) dure trois ans et le deuxième (Matayom Suksa 4 à $6^{7}$ ), également trois ans. Il existe trois domaines d'étude différents au deuxième cycle : sciences, lettres et polytechnique. En lettres, l'élève du premier cycle doit choisir une des langues étrangères proposées par l'école comme sa première langue obligatoire. $\mathrm{Ce}$ n'est qu'à partir de Matayom Suksa 4 (première année du deuxième cycle) que l'apprentissage de deux langues étrangères est permis, la seconde étant facultative.

L'ensemble des deuxièmes langues enseignées en milieu académique, comme matière à option, peut se répartir en quatre groupes : une langue germanique comme l'allemand des langues romanes comme le français, l'italien, l'espagnol, le portugais, des langues asiatiques, principalement le chinois mandarin, le japonais, le vietnamien et le lao ou encore des langues comme le russe et l'arabe.

41 A signaler que malgré l'insistance de la Réforme de l'éducation de 1978 sur l'égalité de toutes les langues étrangères au secondaire, l'anglais reste la première langue obligatoire pour les élèves du premier cycle, alors que le français se classe depuis longtemps comme la seconde langue facultative la plus choisie par les élèves du deuxième cycle.

\section{3. Évolution de la politique éducative officielle face au français}

Dans le passé, l'enseignement des langues étrangères en milieu académique n'exerce qu'un rôle d'instigateur de la culture. De même, on pensait que la connaissance des langues permettait à l'individu de développer son intelligence et d'élargir sa vision. La langue était considérée comme un outil d'accès aux points de vue de l'autre par l'intermédiaire des œuvres littéraires. En ce sens, la compétence en langues étrangères résidait dans la lecture et la traduction des œuvres des grands écrivains. L'apprentissage d'une autre langue était de plus réservé au groupe d'élite tenu pour les savants de la société. Au fur et à mesure que le monde a évolué, l'apprentissage ne visait plus la littérature ou un seul groupe d'apprenants, mais s'étendait à un usage plus grand et un public plus large. La direction choisie s'oriente donc vers la communication et l'échange entre gens de langues et cultures différentes. Il semble nécessaire que les personnes éduquées du XXe siècle comprennent et connaissent d'autres langues que leur langue maternelle.

43 Conscient du grand rôle des langues étrangères dans la vie du peuple thaï, le ministère de l'Éducation a tenté d'adapter la politique linguistique nationale et de modifier le 
système éducatif pour répondre aux besoins des citoyens en voie de modernisation et se préparer à faire face aux nouvelles exigences sociales. Une série de réformes dans l'élaboration des programmes d'études, en fonction des progrès technologiques et des changements sociaux, économiques et politiques du pays, le prouve. La première démarche a été mise en œuvre en 1895 sous la forme du curriculum B.E. 2438. Déjà dans le curriculum B.E. 2471 (1928) a été décrété l'apprentissage d'une première langue étrangère obligatoire et d'une deuxième langue facultative. Partant de cette politique, la plupart des écoles ont toujours proposé aux élèves en lettres d'apprendre l'anglais comme matière principale et le français comme branche secondaire. Cette proposition a rendu le français plus célèbre que d'autres langues étrangères, à savoir l'allemand le chinois ou le pâli. Mais, après la Seconde Guerre mondiale, l'importance du français dans le Sud-est asiatique a diminué, ce qui a abouti au changement du statut du français dans le cursus scolaire. D'après le curriculum B.E. 2498 (1955), le français était dispensé comme une matière à option destinée aux élèves en lettres ou en arts libéraux.

Au début, l'enseignement du français se fait selon la méthodologie traditionnelle qui met le poids sur la connaissance de la littérature et de la civilisation françaises. Puis, l'apprentissage des langues étrangères s'oriente vers des finalités plus utilitaires, du fait que le monde devient plus étroit et que le besoin de communication entre les peuples d'origine différente augmente. Pour cela, l'enseignement a pour but d'élargir les contacts avec les autres. La méthodologie directe est en conséquence bienvenue dans la didactique des langues. Avec cette approche, le français s'apprend sans passer par la langue maternelle, pour habituer naturellement l'apprenant à la langue. Ensuite, la méthodologie audiovisuelle officiellement introduite dans le système éducatif thaï a signifié une importante transition. Le ministère de l'Éducation met en place, dans le Programme d'études de 1975, le remplacement de la méthode traditionnelle par les moyens d'apprentissage audiovisuels. La différence avec les méthodes directe et audioorale, où déjà l'accent a été déplacé de l'écrit et de la grammaire vers les pratiques orales et vers la grammaire inductive implicite, est l'utilisation d'enregistrements et d'images pour présenter des dialogues en langue seconde. L'accès au sens de la parole étrangère se fait à partir de la situation visualisée. Enfin, conscient des besoins des citoyens, le ministère de l'Éducation nationale a introduit dans le programme de 1981 un autre changement de méthodologie. Cette fois, l'approche communicative est mise en œuvre pour répondre aux besoins de type communicatif de l'apprenant. Elle marque une évolution importante dans la didactique des langues : le concept de compétence de communication apparaît pour la première fois. Par ailleurs, ce dernier Programme scolaire national modifie le nombre d'années d'étude du français dans le deuxième cycle du secondaire : trois ans à la place de deux. Toutes les observations qui précèdent n'expliquent qu'en partie la redéfinition des besoins langagiers des apprenants que nous verrons en détail dans ce qui suit.

\section{Présence actuelle du français en Thaïlande}

\subsection{Le contexte social de la langue française en Thaïlande}

45 L'importance des langues étrangères ne cesse de croître. En Thaïlande, par la mondialisation politique, la croissance de l'économie et la multiplication des échanges internationaux, les besoins de connaissances en langues étrangères augmentent progressivement. La maîtrise des langues étrangères assure aux Thaïlandais non 
seulement un outil pour poursuivre efficacement des études supérieures à l'étranger dans leur domaine de prédilection, mais encore un atout important pour être compétitifs sur le marché du travail international. Dans la recherche scientifique et sociale ou dans les échanges internationaux, la nécessité de connaître une, deux, voire même trois langues est indéniable.

Quant au français, quel futur peut-on lui prédire en Thaïlande? Langue particulièrement étudiée dans sa dimension strictement écrite, ne restera-t-il qu'une langue étrangère en option dont l'avantage majeur est d'être un outil menant à gagner des points pour la réussite du concours national d'entrée à l'université ? Répond-il réellement aux besoins de la société comme deuxième langue étrangère, comme langue de communication? Avant d'esquisser quelques réponses, il importe d'examiner de plus près le contexte social thaïlandais dans lequel le français se positionne.

Sur le plan géographique, la distance entre la Thaïlande et la France a des conséquences pédagogiques indéniables. Le contact réel avec le français, langue apprise, ne s'effectue pas régulièrement : séjour quasi impossible dans un pays francophone, interactions verbales très rares avec des natifs, limitation des échanges culturels à travers les mass médias en français, nombre insuffisant de bourses d'études et de stage sont autant de facteurs qui influent directement sur la compétence linguistique de l'enseignant comme de l'apprenant thaï. La séparation géographique rend difficile le perfectionnement linguistique et la maîtrise d'aspects culturels en langue cible. Cette quasi-absence de soutien linguistique est assez puissante pour abaisser à un niveau très bas la motivation à l'apprentissage d'une langue étrangère. L'apprenant ne voit pas l'utilité immédiate que la connaissance du français pourrait lui apporter.

Sur le plan politique, la Thaïlande se distingue de ses voisins étant le seul pays du Sudest asiatique à n'avoir jamais été colonisé. Tout au long de son histoire, la Thaïlande n'a jamais perdu sa singularité linguistique ni sa particularité culturelle. Elle maintient depuis des siècles des traditions, coutumes, rites et rituels qui marquent profondément les mentalités thaïes. Le caractère national thaïlandais s'est développé collectivement en relation avec une seule et unique langue nationale : le thaï standard langue qui unit le peuple thailandais quel que soit son origine. Cet unilinguisme donne à d'autres parlers un statut subalterne et un rôle de véhicule de connaissances et non pas d'outil de communication. Le monolinguisme n'incite pas l'apprenant thaï en langues étrangères à prendre conscience de l'importance ou de la nécessité d'autres langues hors de la vie scolaire.

Sur le plan linguistique, nous faisons l'hypothèse que la distance entre le thaï et d'autres langues étrangères, dont le français, peut freiner l'apprentissage et la motivation de communication. La dissemblance des langues source et cible, les dangers d'interférence et la difficulté des schémas de structuration de la deuxième langue découragent l'apprenant et affaiblissent son espoir de maîtriser la langue non maternelle.

La Thaïlande, pays agricole en voie de développement, mais suffisamment développé pour représenter une zone commerciale intéressante, se trouve en plein essor économique et technologique et poursuit progressivement son industrialisation. Comme dans la plupart des pays en développement, les pouvoirs économiques et politiques sont largement influencés par les États-Unis qui ouvrent la voie de la modernisation et des progrès industriels du royaume. Les Thaïlandais, par conséquent, ont tendance à suivre de plus en plus les modèles techniques, linguistiques et 
socioculturels américains. Le développement dans quasi tous les domaines joue, de ce fait, en faveur de l'anglais. Ce privilège reconnu aux valeurs anglo-américaines pousse les Thaïlandais à apprendre spontanément la langue des anglophones.

51 De même, le facteur économique détermine la place de la langue française dans le royaume de Thaïlande; depuis des siècles, le français occupe la deuxième place derrière l'anglais, étant donné la puissance et l'influence respective des États-Unis et de la France sur l'Asie du Sud-est avant la Seconde Guerre mondiale, aussi bien que leur rôle économique et politique par la suite. Mais, la langue de Voltaire a maintenant tendance à céder sa place à des langues asiatiques, telles que le chinois et le japonais, en raison du rôle significatif que jouent les pays orientaux dans le monde économique et politique actuel. La langue nippone connaît un essor considérable en Thaillande, ce que de nombreux projets de collaboration peuvent expliquer. L'importance de connaître les langues de la région s'intensifie. Sur l'initiative des ministères des Affaires étrangères et de l'Éducation nationale thaïes ${ }^{8}$, la promotion des langues des pays voisins (le vietnamien, le cambodgien, le birman, le laotien et le malais) a été mise en place. Du côté du ministère de l'Éducation, l'enseignement du malais aux étudiants habitant le long de la frontière thaï-malaise ou celui du birman à ceux qui vivent près de la Birmanie font partie du programme d'expansion du choix des langues que les autorités considèrent importantes pour transformer le pays en centre politique, culturel et économique de la région. Dans le même but, le ministère des Affaires étrangères a lancé il y a cinq ans un programme pour former les jeunes Thaillandais dans d'autres langues que l'anglais. Des bourses ont été offertes et deux groupes d'étudiants ont reçu une formation à Hong Kong et Shanghai. Le fait que la demande du pays en matière de langues étrangères a évolué ces dernières années en direction de l'Asie et qu'un nombre réduit d'employés du ministère connaît les langues des pays voisins, impose actuellement à tous les fonctionnaires, nouveaux diplômés, du ministère la connaissance d'une troisième langue étrangère, hors de l'anglais, du français ou de l'allemand. Depuis 1994, plusieurs d'entre eux ont commencé à apprendre les langues du Cambodge, du Vietnam, de l'Indonésie, de la Malaisie et de la Birmanie dans le cadre du programme 'Neighbouring Language Project' du ministère. Parmi ces langues, le vietnamien, l'indonésien et le malais sont les plus populaires en raison de leur écriture basée sur l'alphabet romain.

L'intention d'orienter les jeunes Thaïlandais vers des langues réputées 'socialement utiles' comme le japonais, le chinois et les langues des pays voisins s'explique en termes de meilleure adaptation aux besoins économiques et éducatifs du pays. Il en ressort que l'apprentissage des langues étrangères suit des besoins actuels et urgents, à savoir les relations sociopolitiques avec d'autres pays ou l'adoption de la technologie des pays industrialisés les plus puissants. Cette dynamique économique est génératrice de nouveaux besoins et explique les attitudes évolutives de la jeune génération, c'est-àdire de celle qui apprend les langues étrangères.

Et la langue française, comment se situe-t-elle par rapport aux perceptions et aux attentes des Thaïlandais ? Historiquement, elle est donc bien implantée et répandue dans le Royaume siamois du fait de relations stables entre les deux pays depuis trois siècles. Pourtant le mot "francophonie " n'évoque pas grand-chose pour les Thaïlandais. Il est temps de penser à l'avenir, c'est-à-dire de prendre en compte la nouvelle génération, ses besoins, ses attentes et ses goûts. Tenir compte du profil du consommateur de demain conduit à mieux cibler les stratégies de promotion de la 
langue française. Mais une réflexion sur ce point ne peut faire l'économie d'une approche détaillée quant à la place réelle qu'occupe le français dans le système éducatif thaï.

\subsection{Les objectifs de l'enseignement du français}

54 En vue de bien comprendre la situation actuelle de l'enseignement/apprentissage du français dans la scolarité thaïe, il convient de s'interroger sur les finalités de cet enseignement En principe, l'enseignement mène quelque part. Ce qui est important, c'est autant l'identification des objectifs que leur réalisation.

La Section des secondes langues étrangères du ministère de l'Éducation a défini ainsi les buts principaux de l'enseignement/apprentissage des langues étrangères dans le programme officiel de 1981:

1. Développer les compétences en langues étrangères selon le besoin de l'apprenant ;

2. Acquérir les quatre compétences fondamentales de la langue choisie: compréhension et expression orales et écrites, tout en connaissant la culture des pays qui la pratiquent pour pouvoir communiquer dans cette langue à tout moment ;

3. Employer la langue comme outil pour acquérir des connaissances dans d'autres disciplines, y compris dans l'enseignement universitaire et dans la formation professionnelle ;

4. Apprécier la valeur de la langue choisie et avoir le désir et l'habitude de la lecture.

\subsection{Niveau secondaire}

A la fin du cursus scolaire, l'élève est censé atteindre les buts suivants :

1. Communication à l'oral et à l'écrit en français ;

2. Connaissance de la culture française en comparaison avec la culture thaïe ;

3. Base solide de connaissances pour des études de haut niveau ;

4. Intercompréhension des deux pays ;

5. Application des savoirs théoriques à la pratique professionnelle, à la recherche et aux études dans d'autres domaines.

\subsection{Niveau supérieur}

Après quatre ans de formation universitaire, l'étudiant dont la branche principale est le français est supposé acquérir les compétences suivantes :

1. Communication en français ;

2. Développement des idées et de l'esprit critique ;

3. Ouverture de l'esprit à la culture et à la littérature françaises ;

4. Application du français à des fins professionnelles ou spécifiques ;

5. Bonne base de connaissances pour des études plus avancées ;

6. Préparation au monde de la recherche ou aux professions de haut niveau.

Pour les autres étudiants en français, les objectifs suivants sont fixés :

1. Quatre compétences langagières (écouter, parler, lire et écrire);

2. Formation aux besoins professionnels ;

3. Renforcement de la matière principale ;

4. Enrichissement des connaissances culturelles. 
ogramme officiel, qui favorise l'acquisition d'une compétence et non seulement une connaissance théorique, marque un écart par rapport à l'ancien système. L'accent se déplace du quoi vers le comment. Le but principal de l'éducation est d'offrir à l'étudiant une formation adéquate pour qu'il puisse ensuite, dans sa vie quotidienne, utiliser couramment les langues étrangères apprises. D'ailleurs, conformément à l'orientation plus pratique, utilitaire et sociale des études, la formulation des objectifs assignés aux langues étrangères vise à équiper l'apprenant pour la vie active dans le sens où ce dernier doit être assez compétent pour mettre les quatre compétences en pratique.

\subsection{Le français à l'école secondaire}

le ministère de l'Éducation (avril 1997), la Thaillande compterait 256 écoles secondaires publiques dispensant le français, dont 98 à Bangkok et 158 dans d'autres provinces, ce qui représente $11,33 \%$ du total des écoles publiques du pays. Notons au passage que sur 33 écoles secondaires privées offrant des cours de français, 28 se trouvent à Bangkok.

\subsubsection{Programmes d'études}

\section{Cours offerts aux élèves de lettres au deuxième cycle du secondaire}

Pendant trois ans, les élèves de lettres doivent suivre six cours principaux et un certain nombre de cours complémentaires dont ils choisissent la matière. Les six cours obligatoires ont pour but de faciliter la poursuite des études supérieures. Les cours complémentaires de français fonctionnel, comme celui du tourisme ou du secrétariat notamment, offrent une bonne base à la vie professionnelle. En 3e année, l'élève doit choisir, en plus de sa spécialité, de deux à huit séances hebdomadaires de français par semestre.

\section{Cours de français destinés aux élèves de sciences}

Les cours de français à orientation scientifique sont offerts aux élèves des domaines scientifiques en tant que matière à orientation professionnelle. Cette option libre est possible pour une durée de un, deux ou trois ans. Cependant, l'apprentissage pendant deux ans est conseillé par le Bureau des inspections.

5 Manuels scolaires

Au niveau débutant ou avancé, l'enseignement du français en Thaïlande s'est fondé sur les méthodes de français langue étrangère suivantes :

1955-1969 - Mauger Bleu, En France, A Paris

1970 - Voix et Images de France

1973 -De vive voix

1975 - La France en direct

1981 - La France en direct (nouvelle adaptation)

67 Aujourd'hui, il existe quatre manuels scolaires officiellement approuvés par le ministère de l'Education :

1 -Le Français Élémentaire de G. Mauger et G. Gougenheim

2 -La France en direct de Guy Capelle 
3 -La France en direct (nouvelle adaptation pour la Thaïlande)

4 -Cours de Langue et de Civilisation Françaises de G. Mauger

\subsubsection{Méthodologie(s) d'enseignement du français langue étrangère}

\subsubsection{Au niveau secondaire de la scolarité thaïe} des moyens les plus efficaces pour l'approche de la langue étrangère à partir de l'oral. Voici les objectifs visés alors par le Bureau des inspections pédagogiques pour $l^{\prime}$ enseignement $d u$ français au niveau secondaire ${ }^{9}$ :

1) Pouvoir comprendre, parler, lire et écrire la langue simple de la vie quotidienne ;

2) Pouvoir comprendre et manipuler des structures grammaticales et des phrases apprises sans avoir fait de grammaire explicite ;

3) Pouvoir comprendre la culture du peuple ;

4) Former une connaissance de base de la langue qui permet une étude plus avancée.

En 1975, le manuel La France en direct a été introduit officiellement dans les classes secondaires. A partir de ce moment-là, ce manuel était largement répandu dans les cours de français. En 1981, on a décidé de donner la préférence à l'approche communicative. Ainsi, en 1981, La France en direct de Capelle a été modifié par le Bureau des inspections pédagogiques du Département de l'enseignement général du ministère de l'Éducation, en collaboration avec le Bureau d'action linguistique du Service culturel de l'Ambassade de France en Thaïlande, en vue d'adapter le fichier au contexte local et de répondre aux besoins du public. Soutenu et préconisé par le ministère de l'Éducation, La France en direct (nouvelle adaptation) est actuellement employé comme manuel principal à tous les niveaux des classes secondaires par tout le corps enseignant de l'école publique. Ce manuel adapté souligne la réforme de l'objectif de l'Éducation nationale de 1981 et la politique linguistique du ministère qui mettent l'accent sur l'éducation scientifique et la professionnalisation.

\subsubsection{Au niveau supérieur}

le système éducatif thaï, chaque établissement d'enseignement supérieur est indépendant. Chacun a ses propres traditions et orientations et détermine ses programmes d'études selon ses besoins. Le Bureau des Affaires universitaires se charge de contrôler et d'approuver ces programmes.

71 Après le baccalauréat, l'élève peut continuer ses études dans une université d'État à condition de réussir le concours national d'entrée à l'université. S'il échoue, il peut aller à une des deux universités ouvertes qui permettent aux détenteurs de baccalauréat de s'inscrire sans concours d'entrée. Signalons aussi que les gens exerçant un métier peuvent y reprendre leurs études en raison de l'absence de limite d'âge.

La langue française est enseignée comme branche principale ${ }^{10}$ ou secondaire et comme option dans onze universités.

\subsubsection{Types de français universitaire}

Le français en options de langue et de littérature ${ }^{11}$ 
74 Le premier type d'enseignement constitue l'étude du français à caractère linguistique, littéraire et culturel qui mène à une licence en lettres. Les étudiants de section lettres peuvent choisir le français comme option majeure ou mineure. Les méthodes d'apprentissage sont basées sur l'interprétation et la traduction de textes littéraires et sur la comparaison des registres littéraires entre les deux langues. L'enseignant et l'élève débattent, principalement sur l'étude du style de chaque auteur, en prenant en compte également les influences réciproques entre ce dernier et la société dont il fait partie. Dans ce cas, l'enseignant utilise les textes littéraires comme une source, décrivant la relation qu'entretiennent les textes littéraires avec les significations culturelles. On croyait que l'apprentissage d'une langue étrangère à travers l'étude de la littérature était une méthode permettant de connaître la culture de la nouvelle langue. Le texte littéraire constitue un lieu de rencontre avec l'autre ${ }^{12}$, où les représentations d'une autre culture implicitement véhiculées servent de révélateur d'une image sociale collective. Or, actuellement, quelques professeurs confirment que, dans la lecture des textes en langue étrangère par l'étudiant thaïlandais, c'est l'arrière-plan culturel en langue seconde plutôt que l'ensemble des savoirs sur la complexité lexico-syntaxique qui facilite la compréhension. En d'autres termes, le niveau de complexité linguistique du texte ne suffit pas à lever des obstacles liés à des aspects culturels. Il faut aussi une harmonie entre les prédictions du lecteur et l'information nouvellement reçue. Les enseignants ajoutent qu'acquérir des notions sur la civilisation française transforme positivement les attitudes envers les études de langue étrangère et permet de meilleurs résultats aux examens. A cet égard les connaissances culturelles ne sont pas une conséquence de l'apprentissage de la langue, mais une condition nécessaire pour mieux apprendre la langue.

Remarques sur les programmes : quoiqu'un programme idéal soit élaboré, en réalité un grand nombre de cours ne peuvent pas être dispensés faute de professeurs spécialisés. Dans l'enseignement du français à ce niveau, l'étude de la grammaire et de la littérature est plus répandue que celle de la linguistique "parce que la majorité des professeurs ont rarement des connaissances en linguistique. Ils ont reçu une formation en littérature " (Thip-kong 1994 : 44). Par ailleurs, l'apprentissage se fait de manière plus théorique que pratique. Les cours recommandés n'attirent guère l'apprenant qui préfère en suivre d'autres. Cependant, les universités ne cherchent pas à connaître les besoins des étudiants afin d'organiser les cours en fonction de leurs demandes. Ces derniers sont fixés de façon quasi permanente. L'adaptation du contenu à visée professionnelle n'est pas évidente. En outre, beaucoup d'étudiants remarquent que les cours de civilisation française ont un contenu éloigné de la vie réelle et ne semblent offrir aucune utilité pratique. Sous cet aspect, il nous semble que la pédagogie privilégie la conception de la culture comme un contenu de connaissances plutôt que comme un ensemble de pratiques socioculturelles.

\section{Le français, langue étrangère}

78 L'enseignement de la langue française comme langue étrangère s'adresse aux étudiants qui se destinent à devenir enseignants de français. Ils apprennent les bases de la didactique et suivent un stage professionnel d'un semestre (quatre mois et demi) dans une école du deuxième cycle du secondaire. Ce stage a pour objectif de permettre aux étudiants, dont la plupart travailleront plus tard comme enseignants au secondaire, d'assumer des responsabilités dans l'enseignement, de mener à bien une tâche et de mieux connaître le rôle de l'enseignant et les fonctions de l'organisme éducatif. A ce 
jour, cette formation existe seulement dans trois universités : une à Bangkok et les deux autres en province (Faculté de Pédagogie de Chulalongkorn, de Prince de Songkhla et de Chiang Mai). La formation au niveau de la maîtrise est offerte par deux universités : Chulalongkorn et Kasetsart. des publics intéressés ont incité l'État thaillandais à modifier des méthodes d'enseignement pour mieux les adapter aux attentes sociales. Les objectifs scolaires ont tendance à évoluer vers des formations professionnalisées. Dans cette perspective, l'enseignement du français à objectifs spécifiques vise à répondre non seulement aux besoins de publics variés, mais aussi aux exigences des secteurs professionnels. Vu l'importance que l'état donne à la qualité de la formation, les universités thaïlandaises cherchent à s'adapter en orientant les programmes dans des directions plus spécialisées, accordées au marché du travail.

\subsection{Apparition du français fonctionnel en Thaïlande} de coopération culturelle et technique, qui s'est concrétisé par des bourses d'études et des stages en France et l'envoi d'un nombre exceptionnel de techniciens, scientifiques, médecins, juristes et ingénieurs thaïlandais en France. A cet égard a surgi un problème. 
Presque aucun boursier du domaine technique et scientifique n'avait appris le français. Prenant conscience de cette nouvelle problématique, le Gouvernement français, en collaboration avec le Département de la Coopération technique et scientifique thailandais, a préparé un cours de français fondamental pour débutants dans le but de faciliter à ces boursiers leur premier contact avec des francophones ainsi que l'accès à des sources documentaires écrites en français en fonction de leur spécialité et de leurs besoins d'échanges avec diverses institutions de l'Hexagone.

En parallèle, en raison de l'arrivée d'experts français destinés à travailler en collaboration avec les fonctionnaires thaïlandais, et de l'installation à Bangkok en octobre 1980 du siège de la Communauté européenne pour les contacts avec les pays de l'ASEAN, s'est manifesté un nouveau besoin de personnel qui, non seulement connaissait le français, mais également pouvait l'utiliser en contexte professionnel. L'implantation en Thaillande d'organisations internationales telles que la Croix Rouge Internationale, le Haut-Commissariat aux réfugiés de l'O.N.U, International Relief Organization (I.R.O.), la Thaillande étant devenue un pays d'accueil des réfugiés après la guerre du Vietnam, explique la nécessité d'interprètes, traducteurs ou secrétaires bilingues pour les camps de réfugiés situés dans le Nord-Est et dans l'Est du pays aussi bien que pour les bureaux à Bangkok.

Suite à tous ces facteurs, la question des besoins professionnels a été abordée par l'A.T.P.F. Son séminaire de 1977 a entamé la phase exploratoire de l'enseignement du français fonctionnel. L'idée de réformer l'enseignement du français relève du fait que les cours de français fondamental du niveau secondaire visent simplement à former une base de connaissances pour des études plus avancées et n'aboutissent à aucune formation professionnelle spécifique. Le français a tendance à être plus tard abandonné si les élèves ne s'en servent pas pour poursuivre des études supérieures. Comment assurer un meilleur avenir pour cette langue et susciter une attitude plus favorable des élèves envers elle?

A partir de ce moment-là, les intérêts spécifiques des étudiants de diverses disciplines et non spécialistes en français ont amené les enseignants à diversifier les matières assurées en français. Les objectifs et les contenus ont évolué pour couvrir les besoins d'un public plus large : travailleurs, stagiaires, salariés et étudiants, en respectant le mieux possible les besoins primordiaux de tous.

90 L'expérience du français fonctionnel a été tentée dans un premier temps dans certaines institutions où elle était uniquement destinée aux étudiants en langue possédant une bonne base en français général et envisageant d'élargir leurs connaissances linguistiques à un autre domaine professionnel. A cet égard les matières enseignées ont été en particulier de type professionnel. Plus tard langue " de spécialité » et langue " fonctionnelle » ont commencé à toucher un public désireux d'améliorer sa compétence linguistique en plus de ses connaissances professionnelles. Il s'agit d'un public qui, sans spécialisation en français, a besoin d'avoir accès à des documents à caractère informatif écrits en français. Dans les deux programmes d'études, le français sert d'outil de communication à des fins différentes. Pour les scientifiques, la compréhension écrite reste la priorité. $\mathrm{Au}$ contraire, l'étudiant en lettres vise autant ou même plus le perfectionnement oral que l'écrit. L'apprenant " littéraire " qui est spécialiste en langue tient à compléter son bagage linguistique avec des notions à orientation professionnelle. A ce propos, l'étude de matières dispensées en français « de spécialité » est censée aboutir, d'une part, à la maîtrise de la langue française spécialisée, et d'autre 
part, à l'insertion professionnelle. Dans ce but, l'apprentissage du français à objectifs spécifiques ne concerne pas seulement l'aptitude à communiquer en français, langue de spécialité. En plus d'une langue « instrumentale », le français fournit aussi la possibilité d'accéder au monde du travail. Cela dit, les besoins de l'apprenant dépassent la connaissance théorique et se situent au niveau de la mise en pratique des théories apprises.

Le français "fonctionnel "s'adresse au public des sciences, de la technologie, de l'informatique, de la médecine, de l'ingénierie, de l'industrie et de l'agriculture. Le français s'ajoute à l'anglais, mais il joue un rôle subordonné. Quant au français « de spécialité ", il s'enseigne dans des sections telles que les affaires, le tourisme, le secrétariat, le droit, les affaires internationales où une bonne connaissance en français est indispensable. Les apprenants en français fonctionnel sont débutants en langue mais avancés en matières de spécialité alors que ceux qui étudient le français de spécialité sont débutants dans la matière mais avancés en connaissances linguistiques. Ainsi, une formation professionnelle est donnée à ceux qui disposent d'une bonne maîtrise du français, offrant des enseignements complémentaires, une initiation à une future carrière, qui seront directement utilisables dans le monde professionnel. Dans le cadre des apprenants de spécialité, l'enseignement du français fondamental, cours de langue pour débutants, s'offre pour leur permettre d'acquérir des compétences linguistiques et des compétences professionnelles en parallèle.

\subsection{Enseignement fonctionnel du français ${ }^{13}$}

D'après le programme d'études national de 1976 qui met l'accent sur les besoins professionnels, l'enseignement de la langue française selon des objectifs spécifiques se donne à deux niveaux : débutants du niveau pré-universitaire, étudiants désirant perfectionner leur compétence de la spécialité.

$\mathrm{Au}$ niveau universitaire, les départements de français sont responsables de l'enseignement du français dans des buts spécifiques. C'est la même équipe qui donne les cours de français en options de langue et de littérature. Sur ce plan, deux programmes d'études de français à des fins différentes sont offerts : un programme de langue et un programme professionnel. Comme l'enseignement du français fonctionnel a pour but de compléter une formation linguistique à des fins professionnelles, des cours de spécialisation sont assurés par des professeurs en collaboration avec des intervenants professionnels. Il appartient aux responsables des cours de bien analyser les situations et de prendre des décisions sur la méthode qui assure la réussite.

\section{Le français dans les institutions privées}

\subsection{L'Alliance française}

La plus connue des institutions privées dispensant le français en Thaïlande, est l'Alliance française. Cinq sections se sont installées dans quatre régions : à Bangkok, Chiang Raï, Chiang Maï et Phuket ; celle de Bangkok, située dans les locaux du service culturel de l'Ambassade de France, est le centre administratif ; elle organise des cours de français à tous les niveaux. L'année scolaire se compose de quatre trimestres. Il y a des cours en groupe et des cours privés selon les besoins de l'apprenant. Cette grande 
variété de cours attire chaque année entre 900 et 1200 intéressés. Tous les enseignants sont des natifs de langue française qui sont recrutés localement et les leçons sont données dans leur langue maternelle. L'apprenant a ainsi l'occasion d'apprendre la culture française à travers diverses activités culturelles. Spécialement, les « coursdéjeuner » et " cours-dîner » permettent d'apprendre le français pendant le repas. Cet institut de langue française propose encore des classes en entreprise, des stages spécifiques appropriés aux professionnels ou aux hommes d'affaires. De nombreuses compagnies françaises implantées en Thaïlande recrutent les enseignants de l'Alliance pour donner des cours de français à leur personnel thaï. Actuellement, des cours de conversation par téléphone soit à la maison, au bureau ou en voiture sont monnaie courante.

En principe, les cours destinés aux écoliers, collégiens et étudiants mettent l'accent sur l'enseignement intensif de la grammaire et sur la pratique de la conversation en vue de l'examen d'entrée à l'université ou de l'approfondissement des connaissances avant de poursuivre des études en France. Pour les adultes, les cours portent sur des objectifs spécifiques : français des affaires, français du tourisme et de l'hôtellerie, français du secrétariat et français médical sont offerts sur demande. La formation annuelle des infirmières de Bangkok, qui poursuivent leur stage en France, en est un bon exemple. De plus, les enseignants thaïlandais de français peuvent y suivre des stages de formation continue.

\subsection{Les autres institutions privées}

En Thaïlande, le concours d'entrée à l'université d'État est primordial pour tous les lycéens souhaitant continuer leurs études et avoir un avenir socioprofessionnel prometteur. Beaucoup d'instituts privés poursuivent ces fins ; ils organisent des cours intensifs de rattrapage et de révision de toutes les matières exigées pour les examens. Le sujet le plus important en français réside dans les normes linguistiques. Les questions concernant la civilisation française ou des connaissances générales sont quasi absentes aux examens.

\section{Conclusion}

L'aspect historique et la situation actuelle de la langue française dans le cadre institutionnel thaï ont été présentés de façon descriptive afin que les lecteurs aient un aperçu global de la politique évolutive de ce pays dans l'enseignement des langues. Le contexte thaï, où se présentent des particularités et des spécificités, peut intéresser tous ceux qui font des recherches sur la didactique historique, la politique linguistique et sur les rapports entre langues en contact. La détection de certains blocages qui empêchent la réalisation de certains objectifs scolaires devient un exemple pour l'étude de la problématique de l'enseignement/ apprentissage du français en milieu alloglotte. 


\section{BIBLIOGRAPHIE}

LUANG SITSAYAMKAN (1976). The Greek Favorite of The King of Siam, Bangkok :Chuan Printing Press.

(Second Published)

Bureau de coopération linguistique et éducative, Lettre du BCLE, $n^{\circ}$ 6/mars 1997. Bangkok Post, Outlook le 19 janvier 1998.

Ministère de l'Education, Programme d'étude du français au niveau secondaire 1975.

KITIWATANA, I. (1993). Besoins langagiers et professionnels des enseignants de français langue étrangère dans le secondaire en Thailande. Thèse de doctorat, Université Stendhal Grenoble III.

PORCHER, Louis (1987). Manières de classe, Fenêtres sur cour, Paris : Didier.

THIPKONG, P. (1994). Pour une méthodologie rénovée de l'enseignement du français langue étrangère en Thailande. Thèse de doctorat, Université de Franche-Comté.

YANAPRASART, PATCHAREERAT (2000). Langue et culture dans l'enseignement du français en Thailande, Thèse de doctorat, Université de Neuchâtel.

\section{NOTES}

1. Réf.: The Greek Favorite of The King of Siam (1976).

2. Source : Bureau de coopération linguistique et éducative, Lettre du BCLE, n 6/mars 1997.

3. Langue indo-européenne ancienne, utilisée encore aujourd'hui par les moines bouddhistes du sud de l'Inde.

4. Prathom Suksa 5 et 6 équivaut au CM2 et à la classe de 6e du système éducatif français.

5. L'enseignement des langues étrangères au niveau secondaire est fixé à six cours hebdomadaires de cinquante minutes pendant trois ans.

6. Ce niveau correspond aux $5 \mathrm{e}-3 \mathrm{e}$ du système éducatif français.

7. Matayom Suksa 4 à 6 sont la seconde, la première et la terminale dans le système éducatif français.

8. Source: Outlook, Bangkok Post, le 19 janvier 1998 : 4.

9. Ministère de l'Éducation, Programme d'étude du français au niveau secondaire 1975.

10. Les étudiants en Lettres, Arts libéraux, Sciences humaines, Pédagogie et Education, qui aspirent à la maîtrise du français, ont deux possibilités pour y accéder. Dans l'option « majeure » en français, cette langue est tenue pour matière principale ; dans l'option « mineure », le français reste une discipline secondaire. La différence consiste dans le nombre de crédits pour l'obtention de la licence, variable dans chaque université. En principe, il faut 80-82 unités de valeur pour la majeure et 18-24 unités de valeur pour la mineure. Les études, quelle que soit la branche d'étude, durent quatre ans ou huit semestres au minimum.

11. Cet enseignement a lieu dans plusieurs universités : Faculté des Lettres aux universités Chulalongkorn et Silpakorn, Faculté des Arts libéraux' à l'université Thammasat, Faculté des Humanités aux universités de Chiang Maï, Assomption, Durakijbundit, Kasetsart, Ramkhamhaeng et Srmakarinwirot, Faculté d'Archéologie à l'université Silapakorn, Faculté des Humanités et des Sciences sociales aux universités Prince de Songkhla et Khornkaen.

12. "Produit de la culture, dans les deux sens du terme (" culture cultivée » et culture anthropologique), le texte littéraire retrouve progressivement ses titres de noblesse. Réduit dans un premier temps à $n$ 'être qu 'un support d'apprentissage linguistique ou qu 'une représentation 
factuelle des faits de civilisation, il est actuellement redécouvert comme médiateur dans la rencontre et la découverte de l'Autre. La littérature permet d'étudier l'homme dans sa complexité et sa variabilité. » (Porcher 1987.)

13. Le cours de français «fonctionnel " pour des étudiants venant de secteurs très variés est proposé par sept universités : sections de Lettres à Chulalongkorn et à Assomption, sections artistiques à Silpakorn et à Thammasart, sections de Sciences humaines à Chiang Mai, à Khornkaen et à Prince de Songkhla, sections scientifiques à Prince de Songkhla.

\section{AUTEUR}

\section{PATCHAREERAT YANAPRASART}

Centre de linguistique appliquée, Université de Neuchâtel, Suisse 